\title{
Position and Time System for the RAX Small Satellite Mission
}

\author{
Sara Spangelo, Andrew Klesh,; and James Cutler ${ }^{\ddagger}$
}

\begin{abstract}
This paper presents the Position and Time System (PTS) for the Radio Aurora eXplorer (RAX) small spacecraft. The PTS is a critical mission component that provides accurate temporal and spatial information required for the science objectives. The system faces many challenges due to the restrictions of our CubeSat form-factor and the power, size, and cost limitations. We describe the design approach to selecting the primary PTS components, including the Global Positioning System (GPS) receiver, antenna, and low noise amplifier (LNA). The testing environment, procedures, and performance metrics are described, and results of the PTS performance provided. We discuss the challenges faced in the design and integration stages of the PTS development, and provide important considerations for future missions. Driven by the performance test results, the on-orbit operations procedures are described to maximize our science return while satisfying the mission requirements and constraints. We complete the design cycle by describing how on-orbit data collected using the PTS will be handled and post-processed to provide accurate timing and position information required to satisfy the science objectives.
\end{abstract}

\section{Introduction}

This paper describes the Position and Time System (PTS) for the Radio Aurora Explorer (RAX) satellite developed by the University of Michigan eXploration Laboratory (MXL) and SRI International. RAX is the first CubeSat mission sponsored by the National Science Foundation (NSF) to study space weather phenomena. The position and time data is crucial to the mission's success, providing a common, accurate time source to satisfy the mission objectives. The PTS on a CubeSat is more constrained than on a normal satellite due to the size, mass, and cost limitations that result in fewer feasible options for antenna design and Global Positioning System (GPS) receiver options. Furthermore, attitude control is difficult on a small power-constrained spacecraft that can cause challenges for the PTS.

\section{A. Mission Overview}

RAX is scheduled to be launched as a secondary payload from a Minotaur-IV launch vehicle into a $650 \mathrm{~km}$ altitude, $72^{\circ}$ inclination orbit in Fall 2010. The primary science objective of the mission is to study the formations and distribution of magnetic field-aligned plasma irregularities (FAI) located in the lower ionosphere (at altitudes of $80-400 \mathrm{~km}$ ). ${ }^{1}$ The MXL Team is responsible for designing, building, and testing the spacecraft to support radar receiver experiments in collaboration with SRI International. FAI are studied from orbit since ground radar beams do not always meet the necessary configuration for data collection. The satellite will collect science data from radar backscatter from the FAIs when it passes over designated incoherent scatter radar (ISR) stations, as shown in Figure 1. RAX will then downlink telemetry and payload data when it passes over the SRI and University of Michigan ground stations. RAX is a standard $3 \mathrm{U}$ CubeSat, approximately $10 \mathrm{~cm} \times 10 \mathrm{~cm} \times 30 \mathrm{~cm}$ in size, with mass less than $3 \mathrm{~kg}$. Attitude is determined with magnetometers, an inertial measurement unit, and sun sensors. Passive attitude control is provided by a permanent magnet, which aligns RAX with the Earth's magnetic field, and hysteresis material, which dissipates oscillatory kinetic energy as heat. This passive magnetic control system was designed to ensure RAX antennas are properly aligned for data collection over the experimental zone and communication with ground stations. The experimental zone is a cone centered at Poker Flats in Alaska, and RAX passes through the zone in approximately five minutes during an experimental orbit. The PTS is critical to provide accurate spatial and temporal information for the science mission.

\footnotetext{
*Ph.D. Candidate, Aerospace Engineering, University of Michigan, 1320 Beal Ave, Ann Arbor, MI 48109.

${ }^{\dagger}$ Postdoctoral Researcher, JAXA/JSPEC, Sagamihara, Japan.

${ }^{\ddagger}$ Assistant Professor, Aerospace Engineering, University of Michigan, 1320 Beal Ave, Ann Arbor, MI 48109.
} 


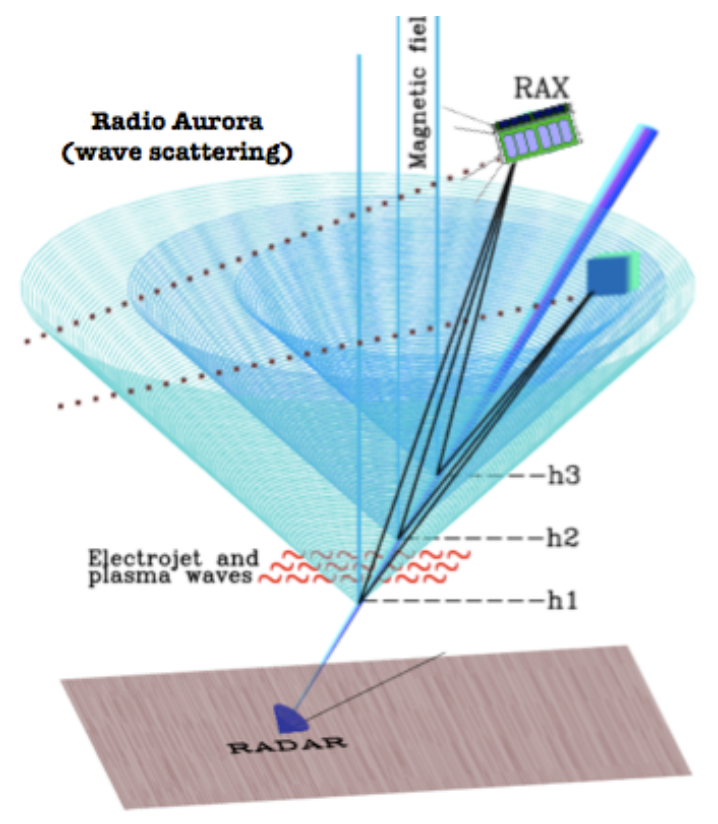

Figure 1. Bistatic radar experiment configuration for RAX mission.

\section{B. PTS Requirements, Constraints, and Challenges}

The PTS provides the spacecraft with a common time source with the required microsecond accuracy for synchronizing ground radar to radar receiver measurements. ${ }^{1}$ The mission requirements state that the PTS must provide satellite location with an accuracy of one kilometer for ranging to the FAIs, as required to achieve the science requirements. The PTS must also satisfy mass, size, environmental, and power constraints specific to the system design. The position and time system consists of a Global Positioning System (GPS) receiver, antenna, Low Noise Amplifier (LNA), and position and time board (PTB). We discuss the requirements and constraints of each component.

For our space usage of GPS, we require the GPS software has disabled the Coordinating Committee for Multilateral Export Controls (COCOM) restrictions, allowing high speed and high altitude operation. In addition, performance will be improved by selecting a GPS receiver which is adjusted specifically for low earth orbit (LEO) applications, in particular with a negative elevation cutoff angle and an extended Doppler window to assist in tracking at high velocities.

The PTS mission requirements will be satisfied if the GPS receiver maintains a continual lock throughout the experimental zone with the GPS satellites. If the GPS receiver fails to maintain lock to a minimum of four GPS satellites, the pulse-per-second (PPS) accurate time signal will drift, resulting in the PTS losing the required accuracy. Proper selection of the GPS antenna and adequate design of the low noise amplifier (LNA) is crucial for attaining the required signal strength such that the PTS maintains lock with the GPS satellites. In addition, sufficient visibility of the GPS satellites requires proper accommodation to ensure sufficient signal strengths are achieved by the GPS receiver.

We must also satisfy the structural requirements of the form-factor according to the CubeSat Design Specification. ${ }^{2}$ RAX will be launched from a standardized CubeSat deployment system, the Pico Satellite Deployer (P-POD). The PPOD is an anodized aluminum rectangular box with a set of torsion springs that open the P-POD door and a main spring mechanism which deploys the satellite. In accordance with the P-POD mechanical requirements, no components may exceed the $6.5 \mathrm{~mm}$ clearance between the CubeSat $(10 \mathrm{~cm}$ square $)$ structure and the inner walls of the P-POD. This means that the GPS antenna size is constrained to a volume less than $10 \mathrm{~cm}$ square and $6.5 \mathrm{~mm}$ tall. The PTS must also be capable of operating in our expected thermal environment between $-40^{\circ}$ and $+85^{\circ} \mathrm{C}$.

Accurate time and position determination is desired for as much of the orbit as possible, even when the satellite is not directly in the experimental zone to enhance the performance of the ADCS and collect data from non-primary ISRs. Constraints on the available power throughout the orbit may limit the ability of the PTS to be powered on and to maintain a continuous lock with the GPS satellites. The available energy is limited by the ability of the spacecraft

\footnotetext{
${ }^{1}$ The PTS also provides information for attitude determination and other secondary mission applications such as evaluating on-orbit determination algorithms, characterizing disturbance forces, and evaluating the performance of the PTS and attitude control system.
} 
to collect and store solar energy from the sun.

With a passively magnetically controlled spacecraft, there are potential problems in maintaining a reliable link with the GPS satellites, particularly prior to the dampening of initial detumbling spin rates and high spin rates about the vector aligned with the magnetic field line. Rapid spin rates without proper attitude control can inhibit meaningful GPS signal acquisition and tracking, because maintaining a constant line-of-sight from the GPS antenna to the GPS satellites becomes more difficult or impossible. Proper attitude control is necessary to avoid rapid tumbling and to obtain meaningful GPS signal acquisition and tracking. The orientation of the spacecraft due to its magnetic stabilization may also present problems, particularly as we pass the South Pole, where our GPS antenna will be pointed towards the Earth (North) away from the GPS constellation.

\section{PTS from Past Missions}

There has been significant work in the design and development of PTS for small and large satellite applications, and the published work has generally focused on specific applications or missions. We present a brief overview of the most pertinent literature in the area of small satellite PTSs, emphasizing the antennas and GPS receivers which have been designed for past small satellite missions.

The challenges of small satellite antenna technology and important interactions between antennas and modern small spacecraft and an overview of existing antenna design options are discussed in Reference 3. Patch antennas, helical antennas, and conical spiral antennas are studied using simulation tools (IE3D) and verified experimentally in a study by Ortigosa. ${ }^{4}$ Due to lack of available surface area for the standard patch antenna, the PCsat spacecraft used a quarter wavelength monopole antenna mounted on the corner of the cubic structure. ${ }^{5}$ The GPS signal acquisition was largely hampered due to the low gain of the antenna and LNA combination, resulting in lower than normal signalto-noise ratios (SNRs). Surrey Satellite Technology Limited (SSTL) and students at the University of Surrey built a miniature $(1 \mathrm{~kg})$ satellite, called Palmsat. The Sarantel helix antenna was chosen due to its compact dimensions and sufficient gain to enable the GPS receiver to achieve accurate position and velocity information at high orbital velocities $(7.5 \mathrm{~km} / \mathrm{s})$. The antenna underwent vibration testing to guarantee it could withstand the conditions it may encounter on-orbit. ${ }^{6}$ Although the design of this PTS is discussed in the literature, flight data was not available, so the on-orbit system performance has not been confirmed.

Cintio studies the TENSOR GPS receiver system designed for PROTEUS, a multi-purpose LEO platform developed by Alcatel Space Industries. ${ }^{7}$ This work studies the link budget, mission analysis, environmental tests and the functional and performance acceptance tests specifically for the first application of PROTEUS, the JASON satellite. The NovAtel OEMV-1-L1 GPS receiver flew on the University of Toronto Space Flight Laboratory (UTIAS) CanX-2 nanosatellite, ${ }^{8}$ where it functioned successfully and was used to collect scientific data. Motivated to reduce the GPS duty cycle due to the power limitations of small satellites while attaining high accuracy position knowledge, Greene et $\mathrm{al}^{8}{ }^{8}$ developed a technique for combining the Simplified General Perturbations Satellite Orbit Model 4 (SGP4) with intermittent GPS orbital information and Two Line Elements (TLEs) from the recent epoch. There are also commercially available specific space-rated GPS receivers for satellite applications; however they are infeasible given the financial limitations of the RAX mission.

To select the components for our PTS, we focused on the antennas and GPS receivers from previous missions in the small satellite community. Many small and large satellites have flown PTSs with varying degrees of sophistication; however few approaches to design, test, and flight verify these complete systems are available in the literature. Our work focuses on describing the procedure for PTS design for the RAX mission, identifying the phases of integration at which different components and capabilities can be tested, and assessing overall system performance.

\section{PTS Design Decisions}

Driven by the science mission, we selected a very simple, cost-effective, low-power, and easy to implement passive attitude control system which would enable the experimental antennas to be orientated correctly to collect science data. The PTS requirements were a secondary consideration, and we designed the PTS based on the constraints of the established control scheme. We now introduce the design drivers, analysis, and decisions of the PTS antenna orientation, antenna type, and GPS receiver and LNA.

\section{A. Antenna Location and Orientation}

The first design decision to consider is the location and orientation of the GPS antenna relative to the spacecraft body. RAX is magnetically stabilized with the permanent magnets aligned such that its long axis $(Z)$ is aligned with the 
Earth's magnetic field lines. RAX is nearly nadir pointing over the experimental zone such that the payload receiver and communication system is pointing towards the Earth, as shown in Figure 2. To maximize the GPS antenna visibility to the GPS constellation while located above the critical experimental zone, it is a natural choice to locate the GPS antenna on the face opposite the communication antennas (the - $\mathrm{Z}$ face). This enables the GPS antenna to be pointed towards the GPS constellation prior to and when directly in the northern experimental zone to aid in achieving a quick lock to the GPS constellation and maintaining lock throughout the critical zone, see Figure 2. Installation of the GPS antenna on the -Z surface avoids any physical conflict with the other antennas and may be done without violating the spacecraft volume constraints, required for storage and deployment from the P-POD used to launch the satellite. Further, this location minimizes potential signal interference from the other receivers and transmitters.

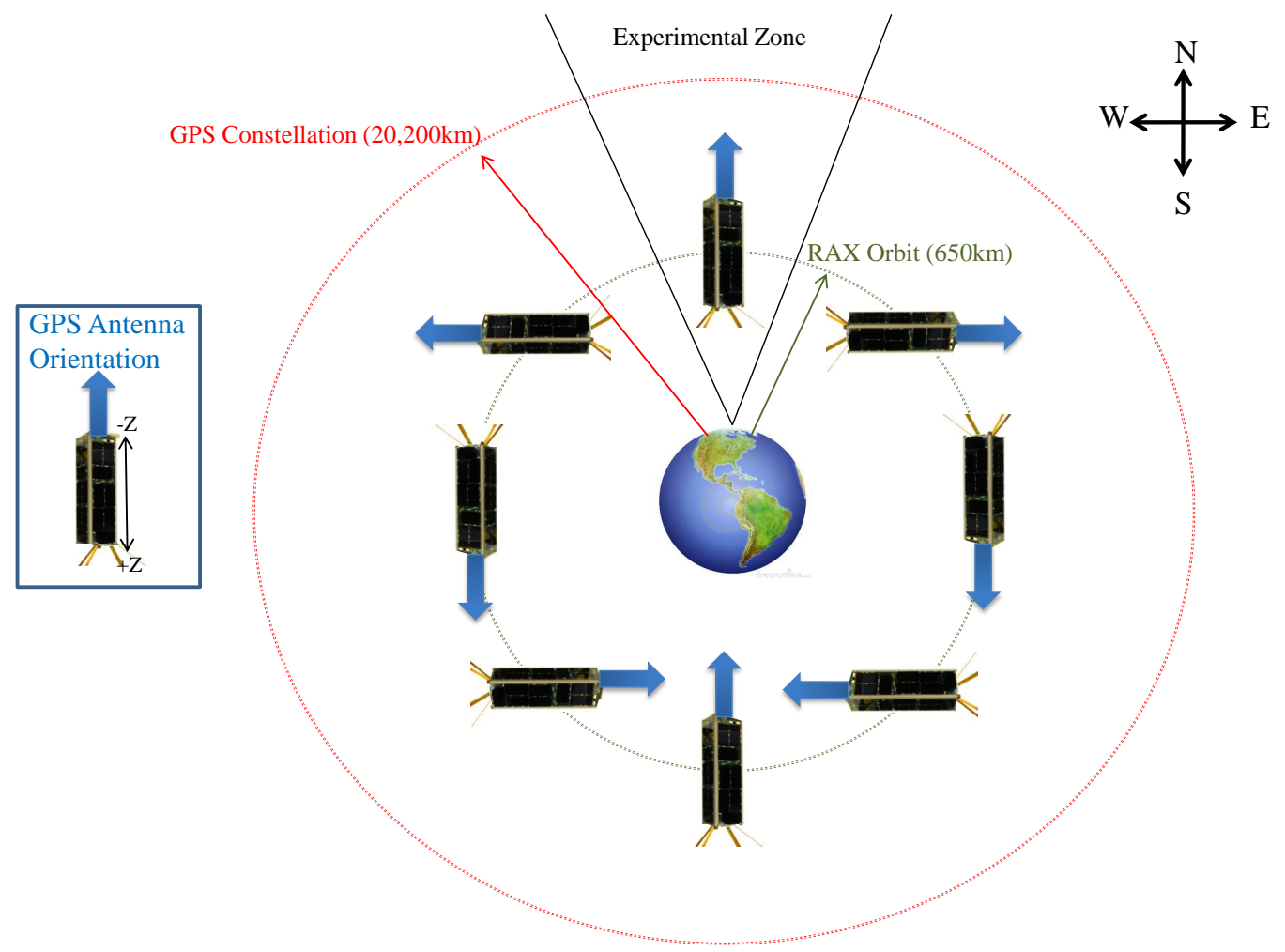

Figure 2. Radio Aurora Explorer (RAX) mission orbit and antenna orientation throughout orbit. Note this schematic is not to scale.

We simulated the number of GPS satellites with line of sight visibility relative to the RAX GPS antenna mounted on the $-\mathrm{Z}$ face of the spacecraft throughout representative orbits. In this analysis, we are assuming the best case scenario that all GPS satellites above the zero degree elevation cut-off are visible. Satellite Tool Kit (STK) and the high fidelity SGP4 orbital propagator were used to generate the orbit information. The number of GPS satellites in view throughout two orbits is shown in Figure 3a), and the average number of satellites in view as a function of latitude is shown in Figure 3b). A minimum of four satellites are required to be visible from the antenna such that the GPS receiver can attain and maintain GPS lock, as shown by the dotted lines in Figure 3. Latitude has a significant impact on the number of satellites in view due to the orientation of the spacecraft throughout orbit (see Figure 2). When RAX is at a latitude lower than $-60^{\circ}$, the number of visible satellites is below the minimum threshold for a significant portion of the sampled latitudes.

\section{B. Antenna Comparison}

The RAX antenna selection must satisfy the highly constrained P-POD form factor in 2 . Several antenna designs were considered, with varying maximum gains, antenna gain patterns, form-factors, and power requirements. We focused our analysis to three types of possible design solutions: helical, monopole, and patch antennas.

The quadrifilar helix antenna is a double loop structure and well-known in satellite communications for its excellent polarization properties. ${ }^{9}$ With a spinning dipole across two orthogonally phased loops, this antenna generates a far 


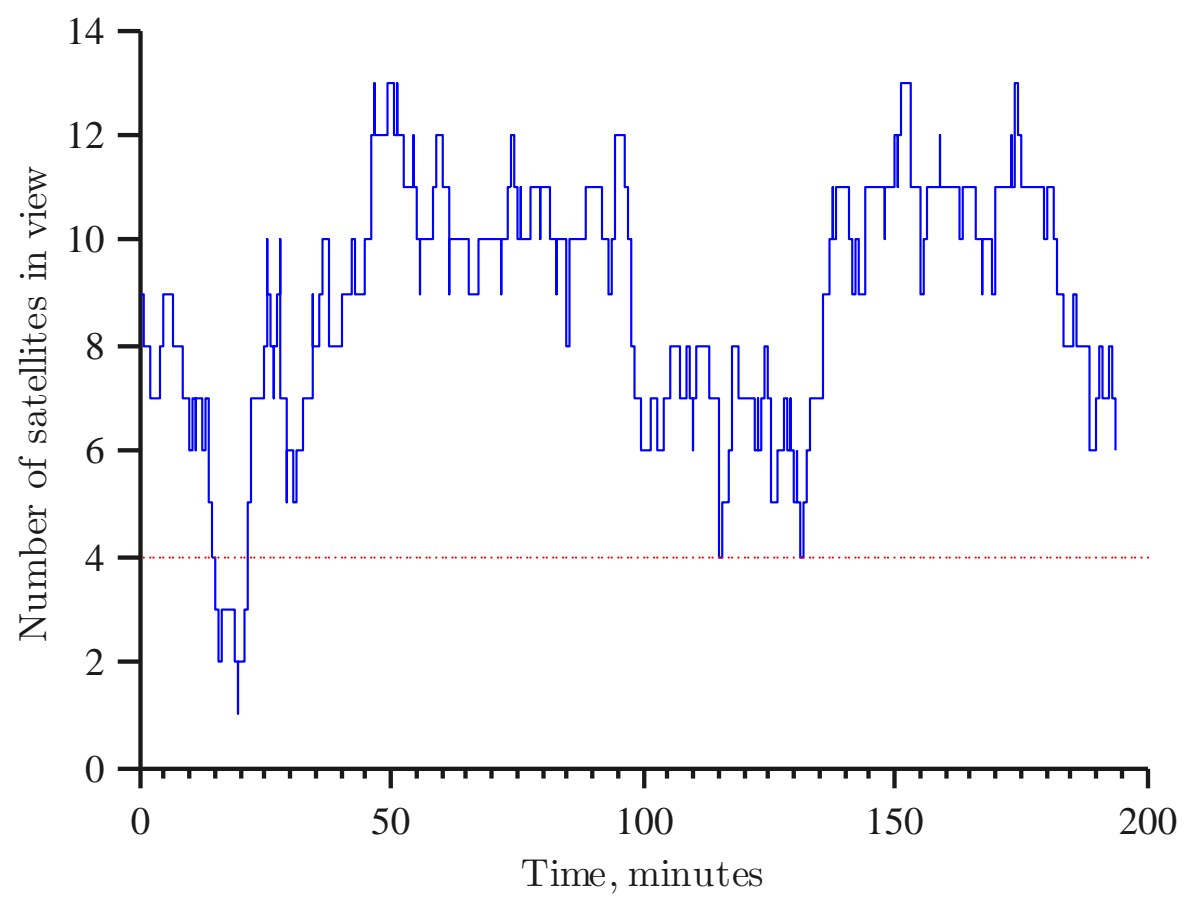

a)

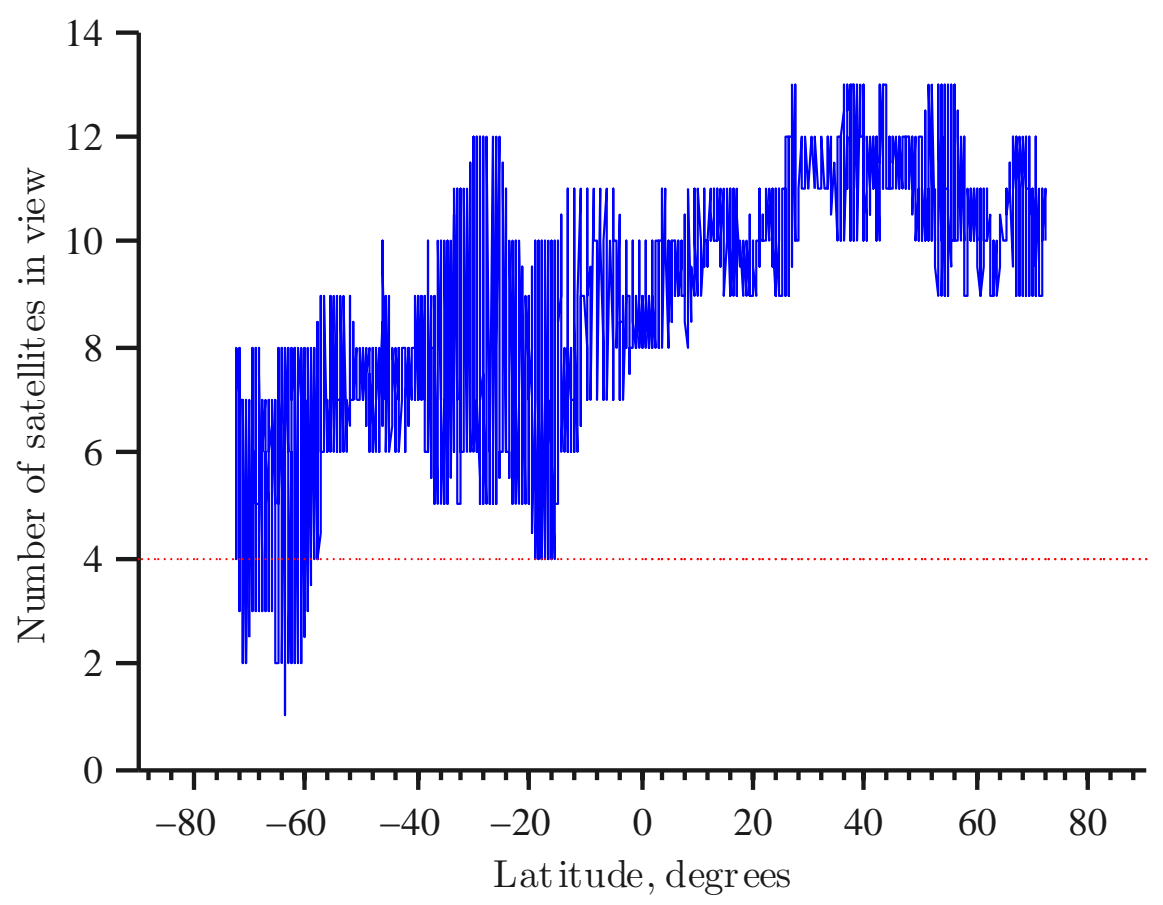

b)

Figure 3. Number of satellites in view for $180^{\circ}$ antenna beamwidth following epoch on September 1, 2010. 5 of 17 
field pattern with a cardioid shape and a broad beamwidth and a null pointing towards the bottom of the antenna. This pattern is ideal for GPS signal reception as the GPS satellites spend most of their viewable time at low elevation angles relative to the antenna. The GPS constellation is at a relatively much higher altitude relative to the spacecraft, therefore when we are at the northern location of our orbit, the null below the antenna is not problematic. However, this does become an issue when we are in the southern part of the orbit. Although the cardioid pattern of a quadrifilar helix has lower peak gain as compared to more directional antennas, the ability to maintain lock with GPS satellites at low elevations is improved. The GeoHelix-S Sarantel helical antenna with a peak antenna gain of $0 \mathrm{~dB}$, typical LNA gain of $+24 \mathrm{~dB}$, and beamwidth greater or equal to $120^{\circ}$ was selected for the Surrey's PalmSat IMAGE mission. The commercially available helical antennas require a deployment mechanism as they do not fit within our off-the-shelf structure. The disadvantages of this system are the required design investment and risks associated with a complex deployment system, particularly problematic given our short delivery timeline.

A monopole antenna is a dipole cut in half with a ground plane backing. Monopole antennas have low gains and are commercially available or may be easily constructed in-house for given applications. The quarter wavelength monopole antenna designed for the PCsat mission provided a roughly toroidal antenna pattern with a sensitivity dip in the bore sight direction, and permitted tracking of GPS satellites even at negative elevations. ${ }^{5}$ The PCsat monopole GPS antenna resulted in overall poor performance related to low GPS satellite SNRs as compared to other antenna types.

The patch antenna, also called a rectangular micro strip antenna, consists of a single metal patch over a ground plane which produces hemispherical coverage. This type of antenna is often covered to protect the antenna structure and has the advantages of being mechanically robust when mounted on rigid surfaces. The Antcom patch antenna satisfies the RAX form-factor and has an integrated active LNA, eliminating the need for investment in the development and accommodation of an LNA elsewhere in the PTS. ${ }^{9}$ The antenna has a typical beamwidth of $103^{\circ}$ to $146^{\circ}$ and is available with an LNA gain of $+33 \mathrm{~dB}$. Some disadvantages include its relatively narrow bandwidth and low efficiency.

\section{Preliminary Design Decisions}

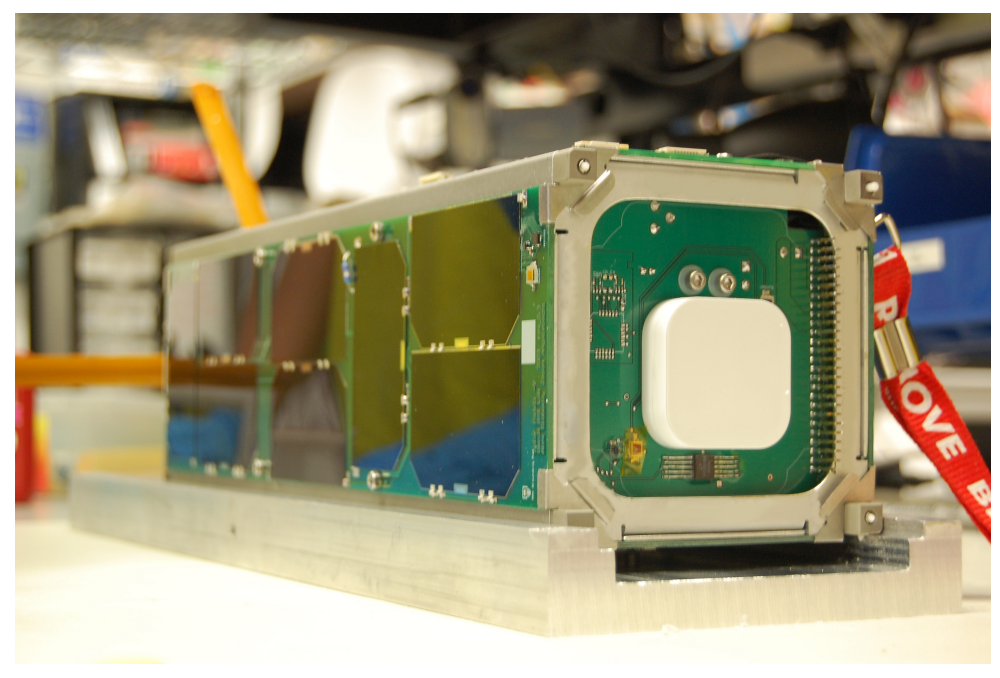

Figure 4. Antcom antenna installed into RAX spacecraft.

The NovAtel OEMV-1-L1 GPS receiver was selected because it satisfies the system requirements and has flight heritage. For terrestrial applications, the receiver manufacturer reports timing accuracy of $20 \mathrm{~ns}$, position accuracy of $0.75 \mathrm{~mm}$ RMS, and velocity accuracy of $0.03 \mathrm{~m} / \mathrm{s}$ RMS. ${ }^{10}$ Furthermore, the form factor is acceptable for our application, the GPS receiver dimensions are $46 \mathrm{~mm} \times 71 \mathrm{~mm} \times 10.3 \mathrm{~mm}$ and its mass is only 21.5 grams.

The Antcom L1 GPS Antenna P/N 1.5G15A with a hemispherical radiation pattern was selected due to its performance advantages over the quarter wavelength monopole antenna and since it satisfied the mechanical requirements without the need for a complex deployment system. The patch antenna is 1.5 " square with a mass of only 42.5 grams. In addition, the Antcom antenna is hermetically sealed and is vibration tested to over $30 \mathrm{G}$ 's. ${ }^{9}$ The antenna has a 
built-in LNA with gain of $+33 \mathrm{~dB}$ to ensure the signal strength requirements are satisfied. The integrated antenna on the RAX spacecraft is shown in Figure 4.

The receiver also requires an interface for power, communications, and other signals and an enclosure to protect against the environment. ${ }^{10}$ The power requirements of the GPS receiver are approximately $1.1 \mathrm{~W}$, and we operate the LNA required for the active antenna is operated at the battery bus $(7.8 \mathrm{~V})$ with a measured current draw of approximately $37 \mathrm{~mA}$. A reliable cable for signal transmission and data communication equipment capable of serial communication are required to interface with the receiver. These requirements are satisfied with a high-quality coaxial cable and the Position and Time Board (PTB) built to house the receiver.

The PTB provides power and communication for the GPS receiver and related systems, and is shown in Figure 5. The GPS receiver is directly mounted to the PTB and encapsulated in an anodized aluminum box for thermal and electromagnetic shielding. The receiver's Universal Serial Bus (USB) connection is accessible and a small breakout port allows direct probing of GPS signals. Primary communication is through a Serial Peripheral Interface (SPI) to Universal Asynchronous Receiver/Transmitter (UART) bridge component that translates the flight computer's SPI interface to the receivers UART interface. The receiver's second UART interface is brought to the main satellite signal bus for monitoring by additional satellite systems. Signal isolators and power control circuitry isolate the PTB when the system is commanded off. A real time clock component provides course timing (ms accuracy) and an auxiliary pulse per second for redundancy. Internal telemetry points are gathered by the flight computer over the internal InterIntegrated Circuit $\left(\mathrm{I}^{2} \mathrm{C}\right)$ bus.

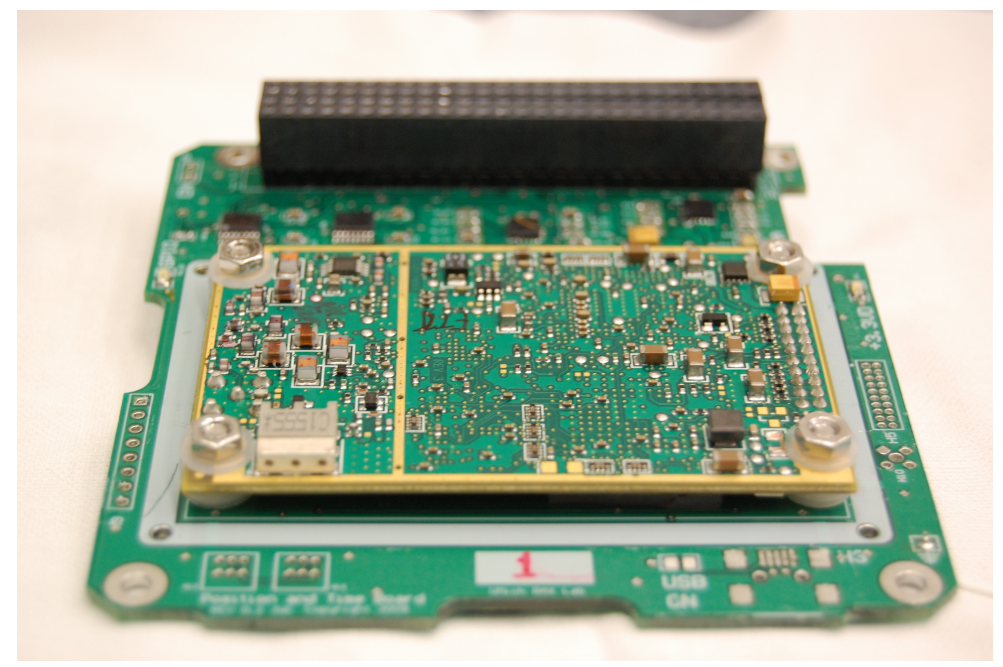

Figure 5. Position and Time Board (PTB) not encapsulated and populated with OEMV-1 GPS receiver for flight. Debugging connectors are not attached.

\section{Testing Environment and Results}

With our final PTS components selected, we proceeded to develop the critical test plan to ensure the full system was functional and to enhance our understanding of its operation. Here we outline the metrics by which we evaluate PTS performance. Due to the importance of component interaction once the system is integrated, we describe three test set-ups at the various stages of system integration we used to test the PTS and describe the results.

\section{A. Evaluating Performance}

We evaluate the performance of the PTS by monitoring the output logs from the GPS receiver, enabling us to examine how the entire system is operating. All of the GPS receiver information we discuss in this section can be commanded to be output from the OEMV-1 GPS receiver in the form of logs at the desired frequency (i.e. once, every 1 second, every 1 minute, etc. $)^{11}$ Most GPS receiver manufacturers describe both the time to acquire a lock upon initial start-up and the accuracy of the receiver, providing the user with baseline expected performance capabilities. The time to acquire a lock is termed the time to first fix (TTFF), and is the time until a location solution is achieved. For our mission, we are also interested in the time until finesteering, when the GPS receiver pulse-per-second oscillator achieves the desired 
accuracy ( $1 \mu s$ for our mission). The start may be considered cold, warm, or hot depending on the conditions upon powering the receiver. In the case where the receiver has no almanac (knowledge of the GPS satellite constellation) or ephemeris (recent receiver position information) and no approximate position or time, the start is considered cold. A warm start is when no recent ephemeris information is available, but the receiver has an almanac stored in the nonvolatile memory (NVM) and an approximate position and time is injected to aid in obtaining lock. The NVM is a type of memory device that retains data when the receiver is power cycled. A hot start is when the receiver has an almanac and a recent ephemeris saved, and an approximate position is injected into the receiver. The receiver is expected to locate and lock onto the GPS constellation more quickly when it has an approximate time and position and an almanac stored on-board. TTFF is improved since the receiver knows which satellites are visible, their elevations, and their Doppler offsets as compared to a start-up where this information is not injected.

The satellite may not lock for various reasons, including if two or more of the satellites are in the same line of sight, satellites are in a cluster formation, or offline for maintenance. Time to finesteering (TTFS) is one of the most critical parameters in PTS performance for our mission, due to our requirement for accurate timing information with minimal energy consumption. In addition, we study other GPS receiver metrics in order to understand whether the GPS receiver is obtaining the appropriate signal strength input and how the receiver is converging to time and position solutions. When commanded, the receiver can output the number of GPS satellites in view, their SNRs, and also their location in the sky (elevation and azimuth). Increased number of satellites in view enhances the GPS receiver's ability to acquire and maintain GPS lock. Since a minimum of four satellites must be in view to obtain GPS lock, this metric is very useful to understand if the PTS is near this threshold and close to obtaining, maintaining, or losing lock.

The signal-to-noise ratio (SNR) provides information about the signal strength of all of the GPS satellites in view of the GPS antenna on-board our spacecraft. We examine the signal-to-noise ratio (SNR) of the on-orbit GPS signal in order to quantify the quality of the incoming GPS satellite data. The GPS receiver manual reports the SNR values are "Poor" if the SNR $<35$, "Average" if the SNR $>35$, and "Good" if the SNR $>45$. We evaluate both the average SNR of all satellites with SNR $>0$ and the average value for the GPS satellites with SNR $>35$ (deemed a normal signal strength) to monitor the GPS satellite tracking.

In the case of cold starts (when a valid almanac is not stored in the NVM), we also monitor the time to acquire a valid almanac. This requires continuous tracking of the GPS satellite constellation to download almanac information, and the manufacturer reports that the OEMV-1 receiver nominally requires 12.5 minutes to download the almanac for terrestrial applications. ${ }^{10}$ Valid almanacs are required to achieve accurate timing information, and furthermore aid the receiver in future hot and warm starts to facilitate expedient acquisition of the constellation.

A pulse per second (PPS) is an electrical signal that precisely indicates the start of a second. PPS signals are output by various types of precision clocks, including GPS receivers. A minimum of four satellites are required to attain lock and achieve the desired level of timing precision for the PTS. When off-nominal situations occur, such as when the temperature of the receiver environment changes or satellites are lost from view, the PPS may drift and introduce error into the time. The drifting characteristics of the OEMV-1 receiver in off-nominal conditions are studied in the upcoming sections.

\section{B. In-house Tests}

We tested the individual components of the PTS, including the GPS receiver, antenna, and LNA, at various stages of integration into the full spacecraft system. Three approaches were taken to test the PTS configuration in-house. First, a fixed terrestrial antenna was mounted on the roof of our building for optimal viewing location relative to the GPS constellation. The antenna was connected to the GPS receiver inside the lab by means of an extended coaxial cable. Secondly, we developed a flexible, portable, and semi-integrated test apparatus consisting of GPS receiver, required coax, antenna with built-in LNA, and an electric power system (EPS). This configuration enabled us to perform outdoor testing in different environments, test different antennas, monitor the effects of the EPS on performance, and study the effects of antenna proximity to our GPS receiver to aid in noise characterization. Finally, we also measured the antenna gain pattern with the fully integrated system in an anechoic chamber.

We tested the performance metrics described in the previous section, including signal acquisition and TTFF for the various start-up conditions, signal strength, and the number of satellites in view. Further, we studied the pulse-persecond drift and the effects of loss of satellite lock and lock recovery by probing the PPS pin on the GPS receiver. Our test results revealed that the GPS receiver operates according to the specifications given for terrestrial applications, and provided us with experience commanding the receiver and interpreting log outputs for the design of our on-orbit operations plan. 


\section{Start-Ups}

The position and time board requires approximately 3-4 seconds to power up and begin to acquire a signal (i.e. search for satellites). Once the unit is powered on, the length of time from the beginning of signal acquisition to the first lock (i.e. tracking of a minimum of four satellites) is the TTFF. GPS satellite lock depends on a number of factors, including proper signal strength and geometry relative to the satellites. In general, large enough signal strength from the spacecraft antenna to at least four satellites is sufficient to achieve GPS lock and be capable of acquiring GPS data (position and time).

The OEMV-1 receiver is reported to have the start-up and reacquisition characteristics in the Table 1 (starting from the time power is supplied to the GPS receiver and active antenna LNA). For terrestrial applications, the OEMV1 receiver is expected to have a 35 second TTFF for hot starts, and one minute TTFF in the cold start case. The manufacturer's reported time to reacquire the constellation, when a position solution has recently been stored in the NVM, is approximately 0.5 seconds. The various types of start-up were tested for the time to first finesteering (TTFFS), and found to be relatively close to the reported values from the OEMV-1 manual. The average results are summarized for the various start-up types in our in-house set-up in Table 1. Our de-integrated system performed slightly worse than the values from the manual. The final integrated system was noted to have performance improvements in cold starts, but slightly delayed TTFFS for hot starts. The final system performance may differ due to the shielding effects of the electromagnetic interference (EMI) material we implemented, see Section IV for a detailed description. The almanac acquisition time is consistent with the expected times from the manufacturer's manual. ${ }^{10}$

Table 1. Average time to first finesteering (TTFFS) and time to first almanac (TTFA) for various stages of PTS integration stages.

\begin{tabular}{|c|c|c|c|}
\hline Test Type & Hot Start TTFFS & Cold Start TTFFS & Cold Start TTFA \\
\hline Reported TTFF (OEMV-1 Manual) & $35 \mathrm{sec}$ & $1 \mathrm{~min}$ & $12.5-15 \mathrm{~min}$ \\
\hline De-Integrated Test (No EMI material) & $46 \mathrm{sec}$ & $2.65 \mathrm{~min}$ & $12.6 \mathrm{~min}$ \\
\hline Integrated Test (EMI material) & $85 \mathrm{sec}(1.42 \mathrm{~min})$ & $1.87 \mathrm{~min}$ & $12.63 \mathrm{~min}$ \\
\hline
\end{tabular}

One option when performing any start is to inject an approximate time and position to the receiver, which is expected to expedite the type to acquire a fix. ${ }^{11}$ When testing this approach, we found that the average time to acquire the finesteering status was not improved. With time and position injection, the receiver required on average 48.3 seconds as compared to only 46 seconds without this injection. In addition, it is possible to manually inject a valid almanac in the case of a cold start, when an almanac is not stored in the NVM. Recent almanacs are readily online from the U.S. Department of Homeland Security ${ }^{12}$ in the form of a text file. Tests in the lab environment showed that injection of an almanac did not expedite the time to finesteering or time to obtain a valid almanac.

\section{Pulse-per-second (PPS) Drifts and Recoveries}

Generally, the period of the GPS PPS clock oscillates within error bounds due to the drifting and the correction steering of the receiver algorithm. We measured this oscillation by comparing two receivers and monitoring the difference between the rising edge of the GPS signal using an oscilloscope. We are interested in characterizing the maximum drift as the PPS oscillates around a nominal signal. In our measurements, we noted an average drift of 10ns and maximum nominal drift of $\pm 30 \mathrm{~ns}$ when the two receivers were in identical conditions and four or more satellites were constantly being tracked and used as part of the GPS solution. This oscillation is lower than our design requirements of $1 \mu \mathrm{s}$. When satellites are lost from the calculation of the solution and fewer than four satellites are in view to the GPS antenna, the PPS naturally drifts and loses precision. We noted that the time signal tended to drift in one direction at a relatively constant rate, so the drift rate is of interest to quantify how the clock error accumulates. We consider an example where we are first tracking the maximum amount of visible satellites (10), and slowly drop out satellites in view but issuing the "LOCKOUT" command, which essentially prevents those satellites from being a part of the solution by de-weighting their range in the solution. The results are shown in Figure 6 . The drift grows significantly and constantly in the same direction after the fourth crucial satellite is lost from the solution, around 14 minutes into the test. The PPS drifts approximately $7.1 \mathrm{~ns} / \mathrm{sec}$, therefore it would require 2.3 minutes to drift $1 \mu \mathrm{s}$.

When the fourth satellite returns to view, the receiver recovers rapidly; the PPS re-attains the signal after approximately 0.1 seconds, almost faster than could be recorded. This rapid recovery is due to the fact that the satellite is still being tracked in the solution just not used in the solution calculation. 


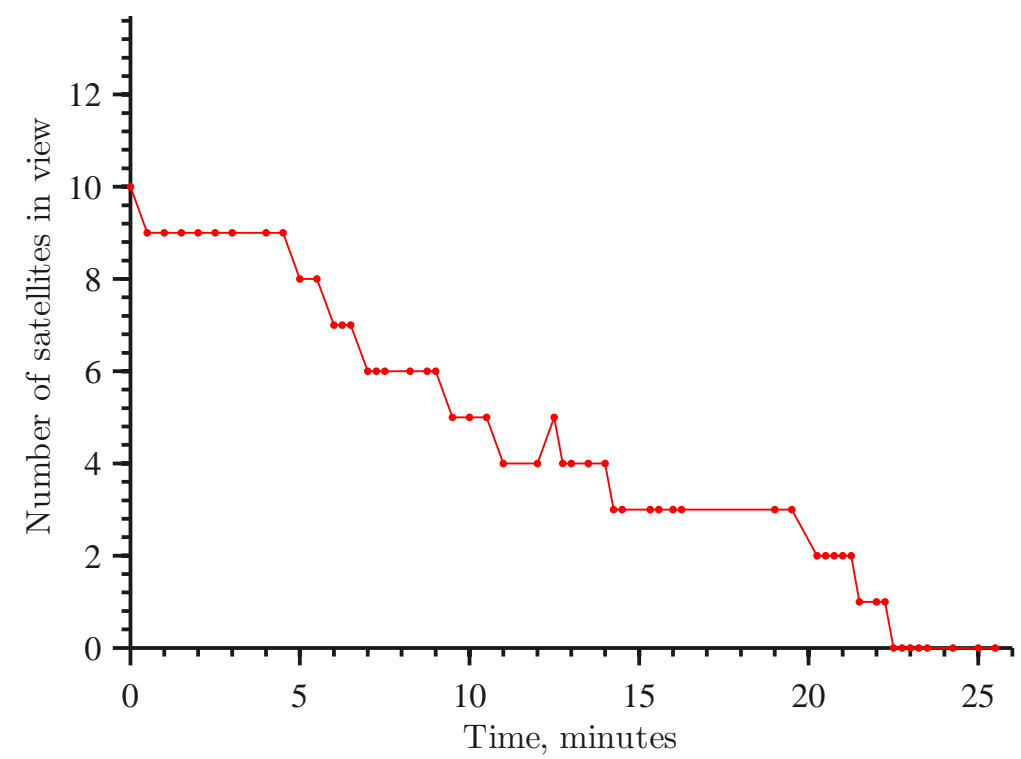

a) Number of satellites used in calculation

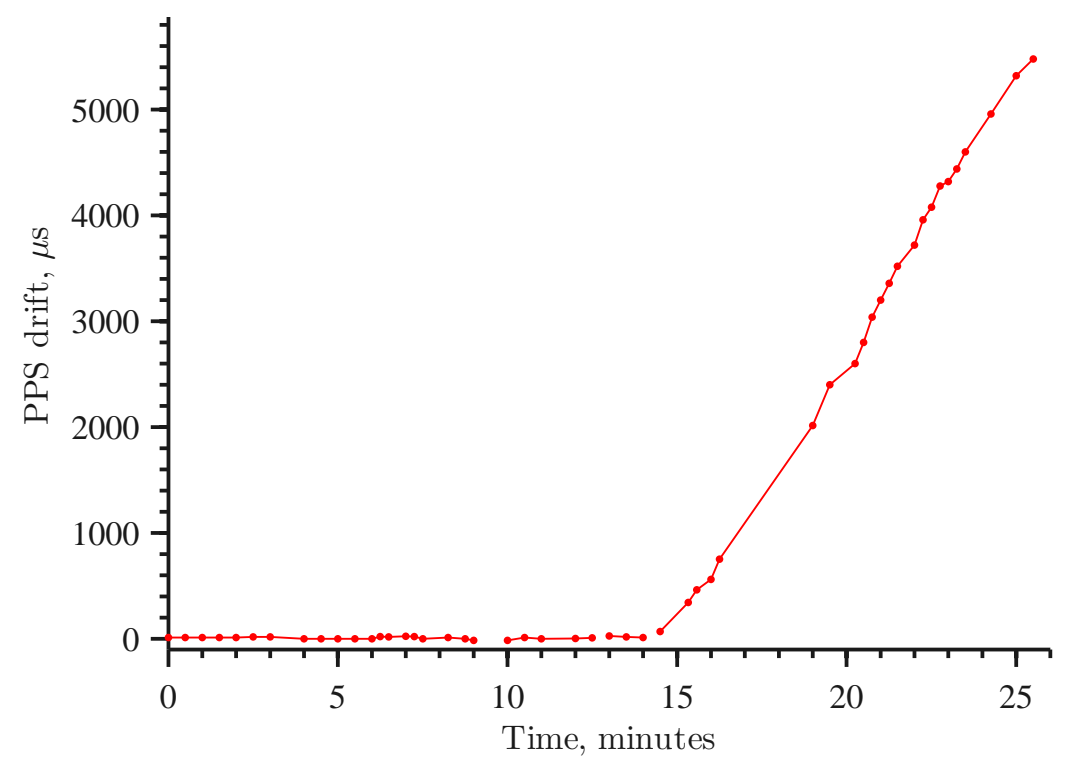

b) Pulse-per-second (PPS) drift

Figure 6. Effect of successive satellites being locked from the GPS calculation. 


\section{Anechoic Antenna Tests}

Antenna performance can be affected significantly due to integration details such as nearby conducting or capacitive loading materials. We therefore collected measurements of the GPS antenna radiation pattern in an anechoic chamber with the fully integrated RAX spacecraft. The results were consistent with the manufacturer's specification and thus appropriate for our design.

\section{Orbital Simulation}

For GPS there is a significant distinction between the space environment relative to the terrestrial one due to the differences in troposphere and ionosphere effects, different elevation viewing angles, and the challenges of tracking GPS satellites at high orbital velocities with Doppler effects. ${ }^{13}$ We are therefore motivated to test our PTS in on-orbit conditions at the representative high altitudes $(650 \mathrm{~km})$ and velocities $(7.5 \mathrm{~km} / \mathrm{s}) \mathrm{RAX}$ will experience. On-orbit simulations have been performed in a GPS Satellite Simulator (GSS) to test the performance of our integrated PTS.

\section{Signal-to-Noise Ratios (SNRs)}

As a baseline, in our terrestrial check-out tests, we found that both the average SNR $>0 \mathrm{~dB}$ and average SNR $>35 \mathrm{~dB}$ was $46.2 \mathrm{~dB}$, meeting the minimum threshold for acceptable signal strength. We have simulated the critical portions of the orbit relative to the GPS constellation consisting of 32 satellites using the GSS. In particular, we studied performance during the start-up phase, when the satellite passes over the experimental zone, and when it passes over the South Pole (due to the worst-case antenna orientation relative to the constellation).

In our simulations, the majority of the orbit had average signal strengths over $45 \mathrm{~dB}$ for the satellites being tracked. In the case of a hot start with no non-essential subsystems on (only the flight computer, PTS, ADCS) the average SNR was $49.5 \mathrm{~dB}$. The SNR values and number of satellites in view drop off when the satellite is in the southern hemisphere and approaching the South pole. Loss of lock occurs when RAX approaches low latitudes (for example, approximately $-67^{\circ}$ in one simulation), when there are no longer the required four satellites in view. This occurs because the satellite is aligned with the Earth's magnetic field vector, thus the GPS antenna is nadir-pointing as it approaches southern latitudes. The latitude at which lock is no longer maintained is largely dependent on the spacecraft's attitude during that orbit. At this point in the orbit, the time status becomes freewheeling, a degradation in time status meaning the position solution is lost and the range bias cannot be calculated. ${ }^{11}$ Once RAX's latitude increases sufficiently, the number of satellites in view and the SNRs recover quickly, and the time status returns to finesteering shortly thereafter. In our simulation, the loss of lock lasts just over 16 minutes (where the full orbit is 97.5 minutes). The latitude where the receiver time and position information is restored is approximately $-18^{\circ}$, a product of the time for the spacecraft to reacquire satellites and compute the solution.

\section{Position Errors}

Uncertainty in our measurement values are important inputs into the Kalman filter, which allows us to make best estimates of our position and attitude required to satisfy the mission objectives (discussed further in Section V). We are interested in the error in the position both when the GPS receiver maintains lock with the GPS constellation and when it loses lock. The on-orbit simulator allowed us to evaluate the error in the receiver output position relative to the simulated spacecraft position and velocity. The norm of the errors were found to be on average approximately 17 meters.

We have also studied the estimated position error output from the GPS receiver in the form of the standard deviation of the Earth fixed Cartesian coordinates output from the BESTXYZ log. The results from the full orbit simulation described in Section 1 are summarized in Table 2. The average standard deviation throughout the entire orbit, the maximum error reported when the receiver loses lock to the constellation, and the average value when a constant GPS lock is maintained are shown for the $\mathrm{X}, \mathrm{Y}, \mathrm{Z}$, and norm of the $\mathrm{XYZ}$ directions. Note that when a lock is continually maintained the average norm error (14.21 meters) is significantly lower than the mission requirements of one kilometer accuracy.

GPS position errors can be caused by a variety of sources, including ephemeris errors ( \pm 2.5 meters), satellite clock errors ( \pm 2 meters), multipath distortion ( \pm 1 meter), and numerical errors ( $\leq \pm 1$ meter). ${ }^{14}$ 
Table 2. Average standard deviations of GPS position output using the BESTXYZ log for one orbit.

\begin{tabular}{c|cccc} 
Magnitude & X direction & Y direction & Z direction & Norm of XYZ \\
\hline Maximum $(\mathrm{m})$ & 78.89 & 63.83 & 228.07 & 249.64 \\
Average with constant lock (m) & 4.34 & 4.58 & 12.74 & 14.21
\end{tabular}

\section{PTS Integration Challenges and Solutions}

Electromagnetic noise and interference is particularly difficult on CubeSat missions given their mass and volume constraints. We define noise as any unwanted perturbations to a desired signal which may block, modify, or interfere with the meaning of the original message. Noise can be injected and dampened in the satellite system by many subsystems and components. It is often difficult or impossible to isolate the noise sources in an integrated spacecraft, as the impact of individual components are often combined in complex ways. Noise can be conducted through power and signal lines, or by radiative means. Note that in this work we do not present a technical analysis of the noise in our system, but a series of observations and inferences which may assist future teams designing small spacecraft.

During early tests on the integrated system, signal acquisition was intermittant. Through a series of systematic de-integrated tests, we determined the cause of the issue was the looped orientation of the coaxial connection between the antenna and receiver. The coaxial cable was found to be at an appropriate length for an efficient loop antenna at the GPS frequency, causing the coax to radiate the signal more efficiently than conducting it to the receiver. We mitigated this issue by switching the locations of the instrument data processing unit (IDPU) and the PTB to accommodate a straight coaxial cable. Figure 7 shows the RAX structure at various stages of integration, where the internal boards are identified in Figure 7c).

Another challenge for GPS operation in our confined embedded system was radio frequency (RF) effects. The GPS receiver is recognized as an RF generating device. The manufacturer advises enclosing the receiver such that it has electromagnetic shielding from hostile RF environments due to nearby transmitters. ${ }^{10}$ In addition, shielding ensures the external subsystems are protected from the effects of the receiver and the system conforms to the RF emissions specifications. To minimize radiated noise, we enclosed the receiver within an aluminum casing and employed electromagnetic interference (EMI) shielding material around the receiver. The interior of the aluminum casing was lined with absorption/reflector material to isolate the receiver from the noise of other subsystems and reflector material was placed on the back end of the patch antenna to aid in reflecting signals away from the antenna.

In the final integrated system, we studied the influence of the individual systems on the GPS performance, including the radios, ADCS, payload, and beaconing radios. Performance, evaluated by the number of satellites in view and their SNRs, as well as the time to achieve finesteering was observed to be independent of the systems turned on and operating.

\section{PTS Operations}

Given the design and testing results, we developed operational techniques for the PTS. Due to the energy limitations of the RAX spacecraft and large power demands of the PTS system, we need to determine the minimal required amount of time the receiver must be powered on to satisfy the mission objectives. Tests on the integrated system revealed that finesteering is generally attained in under two minutes after start-up in on-orbit conditions, indicating that the receiver may be powered on when the satellite is in close proximity to the experimental zone. Based on our test results, we are confident that the PTS will acquire and track satellites and converge to the desired time and position solutions prior to entering the experimental zone. This section describes how PTS data will be collected, handled, and post-processed.

\section{A. Data Collection}

The GPS time and position data is transmitted by the receiver through a serial-to-SPI converter to the main flight processor as it becomes available. As this data time tags the PPS signal, it only needs to complete transmission to the processor within a one second window. This asynchronous approach has the advantage of freeing the processor for more time-critical tasks that require precise interrupts and scheduling. All of the GPS data is logged at the frequency of one Hertz such that the time stamps are synchronized with the PPS signal. ${ }^{11}$

Due to mission requirements and the limitations of the on-board storage capabilities, we must carefully consider the amount and type of data to collect, process, and store. Accurate GPS position and time data is critical for our 


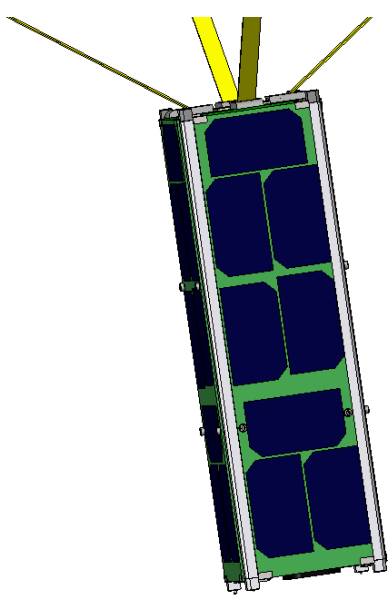

a) Fullly integrated assembly

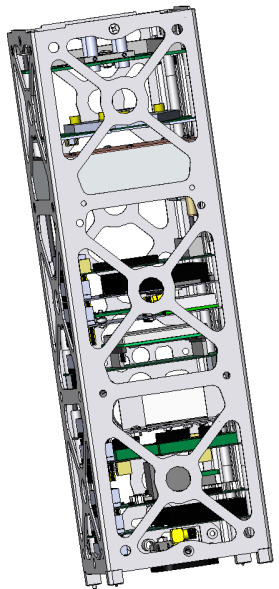

b) Assembly without solar cells and antennas

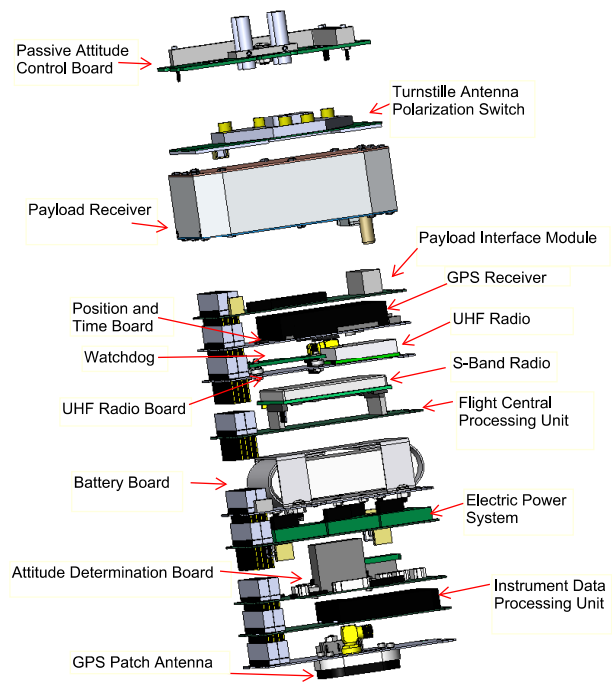

c) Assembly with internal boards only

Figure 7. RAX Computer Aided Design SolidWorks representation 
experiment. Furthermore, status information related to timing, satellite ephemeris, and almanac status is critical in monitoring PTS performance and can be extracted from the GPS log information and would complement our mission. However other mission-critical data, such as data from the on-board radar processor, full-resolution data from science experiments, and ADCS data must also be processed and downlinked. The amount of data we can collect depends on the data downlinking rates and opportunities, and dependent on the individual ground station capabilities and locations. GPS data are text files, and therefore are relatively easy to compress. For example, a typical GPS file can be compressed by a factor of approximately seven using gzip.

There are a multitude of user-specified commands available from the OEMV-1 GPS receiver. We have selected the minimum three sentences required to satisfy mission requirements and plan to log them continuously at a frequency of one Hertz whenever the receiver is powered on. The logs include position information for satellite localization, timing information for the on-board flight computer, and information about the number of GPS satellites in view and quality of the signals. The descriptions and sizes of the logs we select to command are outlined in Table 3. After one orbit of continuous logging, the accumulated GPS data will be approximately $3 \mathrm{MB}$ of data, significant relative to the other sources of data. Therefore, we may only be able to downlink the position and timing information prior to and during the experimental zone, as highly accurate information is critical for mission success during this period.

The GPS log BESTXYZ provides the position solution status and the vehicle position in the WGS84 EarthCentered Earth-Fixed (ECEF) coordinate system. In this coordinate system, the $\mathrm{Z}$ axis is parallel to the direction of the Conventional Terrestrial Pole (CTP) for polar motion, the X axis is the intersection of the WGS 84 Reference Meridian Plane and the plane of the Equator, and the $\mathrm{Y}$ axis completes the right-handed coordinate system. Velocities in the ECEF are also provided, as well as the standard deviation of the position and velocity values. The errors in the values are important inputs into the post-processing Kalman filter (described in Section B) to quantify the errors in our GPS measurements.

Accurate timing information is provided from the GPS receiver using the GPZDA log, which provides Coordinated Universal Time (UTC). Based on the International Atomic Time, UTC has leap seconds added at irregular intervals compensating for the Earth's slowing rotation. Our flight computer requires UTC time as it is the standardized time format in most computer systems, and used to synchronize between the ground stations located in Michigan, Alaska, and California, and the radar transmitter.

The GPGSV log provides information on the number of satellites in view, including their PRN number, elevation, azimuth, and SNR. Note that the receiver may by tracking fewer satellites than are in view, which can be determined by comparing the GPGSV and GPGSA logs. Information on the almanac status can also be extracted from the GPZDA message. The GPZDA log is output from the GPS receiver prior to acquiring a valid almanac, but the UTC time status is set to WARNING as it may not be $100 \%$ accurate. Following the download of the full almanac, the time status reports VALID.

In addition to these nominal logs, we may be interested in commanding additional logs on-orbit for additional position, timing, and PTS performance information. For example, using the ALMANAC command, we can $\log$ the entire GPS navigation message, which is a file of approximately 5.38 kbytes. Due to its large size, we will $\log$ ALMANAC only once every several weeks and store it on-board in case of emergency situations where almanac injection is required. The TIMEA command provides GPS offset time from UTC time and receiver clock offset from GPS time, useful in determining the receiver PPS offset. GPS time is given in the week number since January 5th, 1980, and the seconds into that week, and does not consider leap seconds. We chose to log GPZDA instead of TIMEA as the TIMEA log is significantly larger than the GPZDA one (161 bytes versus 32 bytes), and does not provide the required UTC time directly. Conversions between UTC and GPS time can easily be done on-board the spacecraft or during post-processing on the ground. We may also be interested in logging the CLOCKSTEERING and CLOCKMODEL commands immediately after powering on the GPS receiver, as they provide information on the convergence of the clock model and errors. An additional useful command we plan on using early after the receiver has been powered on is the RANGEGPSL1 command, which provides the number and SNRs of satellites being tracked, and offers the advantage of providing this information prior to achieving finesteering.

All the logs discussed in this section and many others can easily be pre-set or programmed on-orbit using the command architecture described in Reference 11. The desired frequency of each log can also be set by the user, and logs can be cancelled one at a time or collectively. Furthermore, the data stored in the NVM can be reset, including the almanac, ephemeris, last known position, stored commands, and Temperature Compensated Crystal Oscillator (TCXO) offset information. 
Table 3. Description and sizes of GPS sentences logged.

\begin{tabular}{|c|c|c|c|}
\hline Command & Description & $\begin{array}{c}\text { Average size of log } \\
\text { sampled every 1 Hz (bytes/sec) }\end{array}$ & $\begin{array}{c}\text { File size of one orbit, } \\
\text { sampled at 1 Hz (Megabytes) }\end{array}$ \\
\hline GPGSV & GPS Satellites in view & 193 & 1.13 \\
BESTXYZ & Position, velocities and errors & 285 & 1.67 \\
GPZDA & UTC and Time status & 32 & 0.19 \\
\hline Total & All logs & 510 & 2.98 \\
\hline
\end{tabular}

\section{B. Post-Processing}

PTS data is post-processed on the ground using an in-house developed Extended Continuous-Discrete Kalman filter, which takes in GPS and sensor measurements, and using deterministic orbital dynamics and estimates of measurement uncertainty, computes the best estimate of the spacecraft position and attitude. The Kalman filter can take in GPS measurements whenever they are available (for example, at uneven timestamps). We use UTC time for the Kalman filter as it is required for the conversions between the ECEF and Earth Centered Inertial (ECI) frames.

GPS position and velocity data are measured in the ECEF frame, however we must convert these values to the ECI frame prior to implementing the inertial dynamics equations. The Kalman filter is designed to improve the accuracy of incoming measurements using knowledge of the system dynamics, therefore we require that any errors introduced into the model through parameter manipulation and conversions be significantly smaller than the measurement errors themselves. The GPS data is anticipated to have errors less than $<17$ meters, in accordance with our simulated results (Section III). The often accepted single rotation about the Earth's axis of rotation approximation conversion from the ECEF to the ECI frames was not of high enough accuracy for this application. Highly accurate conversions are required to maintain the accuracy of our incoming position and velocity data.

STK is recognized for providing highly accurate position; therefore we first studied their conversion algorithms. Reference 15 provides the conversions between the various STK inertial frame representations available, including the True Equator and Equinox of Date, True Equator and Mean Equinox of Date, Pseudo Earth-Fixed, and J2000, and Earth-Fixed frames. We select the commonly used J2000 inertial frame, and denote the relationship between the inertial and ECEF frames by,

$$
\vec{r}_{J 2000}=P^{T}(t) N^{T}(t) R^{T}(t) W^{T}(t) \vec{r}_{E C E F},
$$

where $P(t), N(t), R(t)$, and $W(t)$ are rotation matrices. $P(t)$ is due to precession, $N(t)$ is due to nutation, $R(t)$ is due to the Earth's rotation relative to an inertial frame, and $W(t)$ is due to the pole wander. Unfortunately the implementation of the rotation matrices for this high fidelity frame conversion is non-trivial.

The AGI orbital experts advised us to use the Standards Of Fundamental Astronomy (SOFA) ${ }^{16}$ open-source code to perform the desired conversion. Although based on different principles, SOFA has been proved to match the STK algorithms to high accuracy. The SOFA rotation between the frames are described in detail in References 17 and 18. We identified and implemented the codes required from their library required for our desired conversion. The naming convention and definitions used by SOFA differ from the STK approach (Equation 1), however mapping between the frames is possible if done cautiously. SOFA considers the International Terrestrial Reference System (ITRS) to be an ECEF frame and the Geocentric Celestial Reference System (GCRS) to be equivalent to an ECEI frame. The appropriate conversions can enable one to convert the ITRS to the GCRS. This includes a conversion from the ITRS to the Terrestrial Intermediate Reference System (TIRS) and then to the Celestial Intermediate Reference System (CIRS), and finally to the GCRS. The GCRS may then be converted to the J2000 frame using a final bias rotation matrix.

The transformation of the velocity from the ECEF to the ECI is slightly more complex than the transformation of the position due to the rotation of the Earth frame relative to inertial space. We assume that the rotation of the ECEF relative the ECEI is constant about the mean rotation axis $(Z)$ of the Earth. Note that this is assuming a single rotation about the $Z$ axis at a constant rate, where precession, nutation, pole wander, and bias effects are neglected. This is a fair assumption given the relative magnitude of the rotation matrix $R(t)$ relative to the other rotations influencing the conversion. The angular velocity is expressed,

$$
\omega_{F / I}=\left[\begin{array}{lll}
0 & 0 & \omega_{E a r t h}
\end{array}\right]^{T},
$$

where the subscript $F / I$ denotes the ECEF frame relative to the ECEI frame. The rotational rate $\omega_{\text {Earth }}$ is based on the rotation of the Earth relative to the Inertial frame, and can easily be determined using the expression provided in 
Reference 17. A simpler approach includes considering the expression for the Greenwich (Mean) Sidereal Time. ${ }^{19}$

$$
G M S T=18.697374558+24.06570982441908 \cdot D,
$$

where GMST is expressed in hours and decimal parts of an hour, $D$ is the interval in days including any fraction of a day since 2000 January 1, at $12 \mathrm{~h}$ UT (interval counted positive if forwards to a later time than the 2000 reference instant), and the result is freed from any integer multiples of 24 hours to reduce it to a value in the range $0-24$. This approach is accurate to within 0.1 second per century. The angle and rotational rate are,

$$
\begin{gathered}
\theta_{\text {Earth }}=2 \pi\left(\frac{G M S T}{24}\right), \\
\omega_{\text {Earth }}=2 \pi\left(\frac{24.06570982441908}{24}\right),
\end{gathered}
$$

where $\theta$ is in radians and $\omega_{\text {Earth }}$ is in radians/day.

Using the transport theorem, the velocity in the ECEF frame is expressed,

$$
\begin{aligned}
& \left.\stackrel{\vec{x}}{\vec{x}_{\mathrm{B} / \mathrm{O}}}\right|_{I}=\left.\overrightarrow{\vec{x}}_{\mathrm{B} / \mathrm{O}}\right|_{I}+\omega_{F / I} \times\left. x\right|_{I}, \\
& \left.\stackrel{\mathrm{I}^{\bullet}}{\mathrm{B} / \mathrm{O}}\right|_{I}=\left.R_{I / F} \stackrel{\overrightarrow{\mathrm{F}}^{\bullet}}{\mathrm{B} / \mathrm{O}}\right|_{F}+\omega_{F / I} \times\left(\left.R_{I / F} \cdot x\right|_{F}\right),
\end{aligned}
$$

where the positions and velocities are expressed in the body frame relative to the common origin of the ECEF and inertial frames, $(B / O) .\left.x\right|_{I}$ is the position expressed in the ECI frame, $\left.x\right|_{F}$ is the position expressed in the ECEF frame, $\left.\stackrel{\mathrm{I}}{x}_{\mathrm{B} / \mathrm{O}}\right|_{I}$ is the velocity relative to the ECI frame expressed in the inertial frame, $\left.\overrightarrow{\vec{x}}_{\mathrm{B} / \mathrm{O}}\right|_{I}$ is the velocity relative to the ECEF frame expressed in the inertial frame, $\stackrel{\overrightarrow{\mathrm{F}}_{\bullet}}{\vec{x}} \mathrm{~B} /\left.\mathrm{O}\right|_{F}$ is the velocity relative to the ECEF frame expressed in the ECEF frame, and $R_{I / F}$ rotation matrix from the ECEF to the ECI frame.

We tested the frame conversion using ECEF Cartesian position and velocities available from STK, converting them to ECI using our algorithm, ${ }^{19}$ and then comparing the results to the ECI STK (ICRF frame) values at the same time step. The errors in our verification were $<4 \mathrm{~m}$ in position and $<30 \mathrm{~cm} / \mathrm{s}$ in velocity, which are considerably smaller than the expected error with the GPS position and velocity, and therefore acceptable for our implementation.

\section{Conclusions and Future Work}

The PTS is critical for success of the RAX science mission. This paper describes the design and testing of the PTS components at various stages of system integration. We have outlined the design methodology of the PTS driven by the science requirements, control system, and structural limitations of the mission. Previous missions, simulations, analytic analysis, and in-house testing have driven the design decisions for the GPS receiver, LNA, coaxial cable, and integration into the spacecraft. We have thoroughly described our testing environment and methods of evaluating system performance. Preliminary tests led us to make PTS design modifications and also helped drive the on-orbit operational planning. The mission operations, GPS data handling and post-processing to satisfy the science objectives are described. Future work will aim to extend our understanding of the PTS performance. We will analyze onorbit GPS data to aid in assessing system performance and compare these results to the expected performance based on terrestrial and simulation tests. The results of this work will provide feedback for future PTS developed at the University of Michigan and the small satellite community at large.

\section{References}

${ }^{1}$ T. Moretto. Cubesat Mission to Investigate Ionospheric Irregularities. In Space Weather: The Journal of Research and Applications, November 2008.

${ }^{2}$ Cubesat Design Specification. The CubeSat Program, Cal Poly SLO, August 2009.

${ }^{3}$ S. Gao, K. Clark, M. Unwin, J. Zackrisson, W.A. Shiroma, J.M. Akagi, K. Maynard, P. Garner, L. Boccia, G. Amendola, G. Massa, C. Underwood, M. Brenchley, M. Pointer, and M.N. Sweeting. Antennas for modern small satellites. IEEE Antennas and Propagation Magazine, 51(4), 2009. 
${ }^{4}$ Juan I. Ortigosa, Neus Padros, and Magdy F. Iskander. Comparative Study of High Performance GPS Receiving Antenna Designs. volume 3 , pages 1958 - 1961, Baltimore, MD, USA, 1996.

${ }^{5}$ S. Leung M. Markgraf, O. Montenbruck. A Flexible GPS Tracking System for Sub-orbital and Space Vehicles.

${ }^{6}$ Sarantel GPS Antenna to Debut in Space on Surrey Satellite Technology's New Miniature Satellite. Sarantel Website, http://www.sarantel.com/press.

${ }^{7}$ A. Di, Cintio and G. Adami. Results of the Qualification and Validation of a GPS Receiver for a Small Satellite Mission. Number 457, pages 287 - 298, Montreal, QUE, Canada, 2000.

${ }^{8} \mathrm{M}$. Greene and R. Zee. Increasing the Accuracy of Orbital Position Information from NORAD SGP4 Using Intermittent GPS Readings. In Conference on Small Satellites Proceedings, August 2009.

${ }^{9}$ The Right Antenna Makes GPS Work. Microwave Product Digest, http://track.sfo.jaxa.jp/spaceops98/paper98/track2/2b002.pdf.

${ }^{10}$ NovAtel. OEMV Family Installation and Operation User Manual. OM-20000093 Rev 8, Aug 2008.

${ }^{11}$ NovAtel. OEMV Family Installation Reference Manual. OM-20000094 Rev 6, Aug 2008.

${ }^{12}$ Navigation Center, U.S. Department of Homeland Security. http://www.navcen.uscg.gov, July 2010.

${ }^{13}$ Gustavo Baldo Carvalho, Per Gustavsson, Stephan Theil, and Helio Koiti Kuga. Standard Ionospheric and Tropospheric Models for GPS on Aerospace Applications: Geometrical Extension. volume 11, pages 7262 - 7272, Valencia, Spain, 2006.

${ }^{14}$ G. Gerten. Navigation Modeling Operations Space, Control, and User Segments. User Manual, Jan 2007.

${ }^{15}$ Earth Coordinate System. Satellite Tool Kit (STK) Internal Document, July 2007.

${ }^{16}$ International Astronomical Union. Standards of Fundamental Astronomy (SOFA) Tools for Earth Attitude, December 2009.

${ }^{17}$ IERS Technical Note No. 32. International Earth Rotation and Reference Systems Service (IERS), 2003.

${ }^{18}$ Dennis D. McCarthy and Gerard Petit. IERS Technical Note No. 21. International Earth Rotation and Reference Systems Service IERS, July 1996.

${ }^{19}$ P. K. Seidelmann. Explanatory Supplement to the Astronomical Almanac, 1992.

${ }^{20}$ Derek Dalle. Standards Of Fundamental Astronomy (SOFA) Tools for Earth Attitude in MATLAB. http://aeropegasus.engin.umich.edu/gitweb, June 2010.

\section{Acknowledgements}

We would like to thank Dr. Eric Gustafson, Matt Bennett, and Stephanie Gowell for their input and support. Thanks to Ben Kempke and Eric Wustrow for their flight computer and data handling expertise and the entire University of Michigan RAX Team. We also recognize the contribution of Derek Dalle, who converted the original SOFA C code functions into MATLAB, and made it publicly available. ${ }^{20}$ We would also like to acknowledge the AGI Support for their valuable insight and knowledge. Finally, we would like to acknowledge Orbital Sciences Corporation and The Aerospace Corporation for their valuable help and guidance. 